\title{
Ecthyma gangrenosum caused by Stenotrophomonas maltophilia in a neutropenic leukaemic infant: A case report
}

\author{
D K Das, ${ }^{1} \mathrm{MD}$ (Paed), DCH; S Shukla, ${ }^{2} \mathrm{MD}$ (Paed) \\ ${ }^{1}$ Department of Pediatrics, Hi Tech Medical College, Bhubaneswar, Odisha, India \\ ${ }^{2}$ Department of Pediatrics, SCB Medical College, Cuttack, Odisha, India
}

Corresponding author: D K Das (dr.dillipdas@gmail.com)

\begin{abstract}
Ecthyma gangrenosum (EG) is a cutaneous lesion, mostly caused by pseudomonas in immunocompromised patients. Other bacterial and fungal pathogens have also been reported. It can occasionally affect previously healthy children. The cutaneous findings are characterised by small indurated papulovesicles, progressing rapidly to necrotic ulcers with surrounding erythema and a central black eschar. Sites most commonly involved are the buttocks, perineum, limbs and axillae; the face is less commonly involved. We are presenting a rare case of EG in a neutropenic infant who had just completed the induction phase of chemotherapy for acute lymphoblastic leukaemia. The gangrenous lesion was on the face involving the tip of the nose, which is an uncommon location. Blood and pus cultures grew Stenotrophomonas maltophilia, which is a rare cause of EG. The patient was treated with IV antibiotics (colistin for 14 days) and improved.
\end{abstract}

S Afr J Child Health 2016;10(1):99-100. DOI:10.7196/SAJCH.2016.v10i1.957

Ecthyma gangrenosum (EG) is a welldescribed skin lesion, mostly seen in immunocompromised patients. The lesion characteristically progresses rapidly from small indurated papulovesicles to necrotic ulcers with surrounding erythema and a central black eschar. ${ }^{[1]}$ Although rare, the presence of EG is indicative of severe systemic infection with a potentially fatal prognosis. It typically occurs on the extremities or in gluteal and perineal regions. Although Pseudomonas aeruginosa is the pathogen most often associated with EG, other organisms have also been reported. ${ }^{[2]}$ We report a case of EG caused by Stenotrophomonas maltophilia, presenting as an aggressive necrotic facial skin lesion involving the tip of nose in a 9-month-old boy who was receiving treatment for acute lymphoblastic leukaemia (ALL).

\section{Case}

A 9-month-old boy with ALL who had recently completed the induction phase of chemotherapy, presented to Hi Tech Medical College, Bhubaneswar - a tertiary care hospital in the eastern part of India - with complaints of fever for 3 days and a gangrenous lesion (which started as vesicle and progressed rapidly to gangrene) on the nose for 2 days. Prior to the diagnosis of ALL, he had been well, without any major illness. Examination revealed mild anaemia, and no lymphadenopathy. Examination of the nose showed a brownish black gangrenous ulcer surrounded by an erythematous halo (Fig. 1). Haematological investigation showed a leucocyte count of

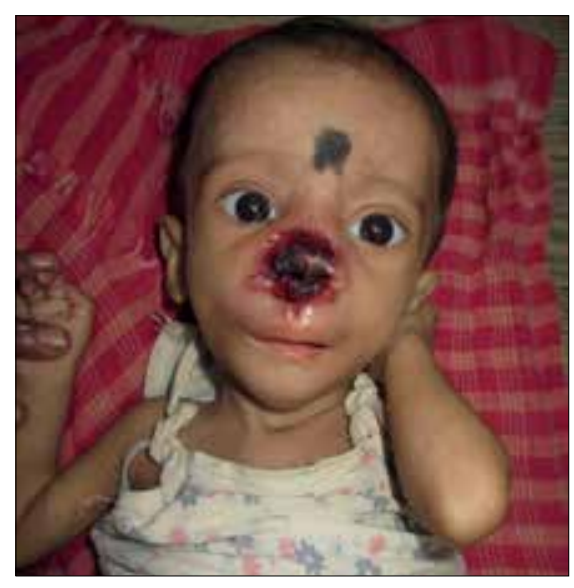

Fig. 1. Tip of the nose showing brownish-black gangrenous ulcer surrounded by an erythematous halo suggestive of EG.

$1200 / \mathrm{mm}^{3}$ with an absolute neutrophil count of $380 / \mathrm{mm}^{3}$. C-reactive protein was $80 \mathrm{mg} / \mathrm{L}$. Blood and pus cultured from the site showed growth of S. maltophilia. A diagnosis of EG caused by $S$. maltophilia was made. Empiric antibiotic therapy consisted of ceftazidime and amikacin intravenously, but this was changed to colistin based on sensitivity results conducted on the isolate. Colistin was continued for 14 days and the lesion healed completely. $\mathrm{He}$ was well at discharge 21 days after admission.

\section{Discussion}

EG generally develops in patients with underlying immunodeficiency, but occasionally can occur in immunocompetent individuals. ${ }^{[2]}$ Factors that are associated with higher mortality include neutropenia, septic shock, inappropriate or delayed antibiotic therapy and resistant microorganisms. ${ }^{[2,3]}$ EG has also been described in infants and young children with transient risk factors, such as concurrent viral infection and recent antibiotic therapy. ${ }^{[4]}$ EG is caused by invasion of microorganisms into the media and adventitia of subcutaneous vasculature, precipitating a haemorrhagic occlusive vasculitis. ${ }^{[5]}$ The skin lesions of EG are the manifestation of this necrotising vasculitis. The lesions characteristically begin as an erythematous nodule, macule, vesicle or bulla and evolve into gangrenous ulcerations with a black eschar and a surrounding rim of erythema. ${ }^{[6]}$ The vesicles, initially filled with serous fluid, appear on the surface of the oedematous skin, and then coalesce to form large bullae that slough away, leaving ulcerated, necrotic centres with erythematous halos. ${ }^{[6,7]}$ Lesions progress very rapidly. The gluteal region is most commonly involved ( $57 \%$ cases), followed by extremities including the axillae (30\%) and the trunk (6\%). The face is also affected in $\sim 6 \%$ of cases. ${ }^{[7]}$ Fever and other constitutional symptoms may be present, depending on the extent of the underlying infection and the patient's immune status. Gastrointestinal and respiratory complaints are also commonly described. ${ }^{[8]}$ Suspicion for EG warrants a prompt diagnosis with cultures and sensitivities performed on blood and pus swabs or tissue specimens.

$P$. aeroginosa is the most common offending organism. Other organisms which have been isolated in patients with EG are shown in Table 1. 
Table 1. Organisms isolated in patients with EG

\begin{tabular}{lll}
\hline Gram-negatives & Gram-positives & Fungi \\
\hline Escherichia coli & Staphylococcus aureus & Aspergillus fumigatus \\
Klebsiella pneumoniae & Streptococcus pyogenes & Candida albicans \\
Morganella morganii & & Fusarium solani \\
Neisseria gonorrhoea & & Meterhisium anisopliae \\
Serratia marcescens & & Mucor pusilus \\
S. maltophilia &
\end{tabular}

S. maltophilia is an aerobic, Gram-negative bacillus and mostly causes opportunistic nosocomial infection. ${ }^{[9]}$ Colonisation with S. maltophilia is most commonly encountered in patients with immunosuppression. Skin and soft-tissue infection in the form of cellulitis, infected mucocutaneous ulcers, EG and paronychia have been associated with $S$. maltophilia infection. ${ }^{[9]}$ The route of transmission is unknown; it is speculated that invasion may take place via defects in mucous membranes and by colonisation of central venous catheters. Infections with $S$. maltophilia are often lifethreatening because of intrinsic resistance to many antibiotics and the general condition of the affected patients. ${ }^{[9]}$

Early diagnosis and prompt treatment of EG are crucial for decreasing mortality and preventing complications associated with longterm sequelae. The choice of antimicrobial treatment depends on the site, severity of infection and antimicrobial sensitivity tests. Initial antibiotics should cover the Gramnegative organisms. Commonly used antibiotics are ceftazidime, carbenicillin indanyl sodium, gentamicin sulfate, imipenem, mezlocillin and piperacillin sodium. A combination of an antipseudomonal $\beta$-lactam agent and either an aminoglycoside or a quinolone is used for empiric therapy. Once culture reports are available, antibiotics should be modified accordingly. ${ }^{[10]}$

Trimethoprim-sulfamethoxazole (TMPSMX) is recommended as the agent of choice for treatment of S. maltophilia infection. Ticarcillin-clavulanate has good activity against S. maltophilia and is the agent of choice in individuals intolerant of TMPSMX, or if the organism is resistant to TMPSMX. Alternative antibiotic agents that may be used to treat isolates resistant to first-line agents include colistin and polymyxin $B$.

Surgical drainage of localised abscesses and debridement of all necrotising tissues may be needed to prevent the spread of infection and septicaemia. Large tissue defects may require reconstructive surgery.

\section{Conclusion}

Our case illustrates an unusual presentation of EG, both in terms of the site involved and organism isolated. Patients presenting with EG warrant hospitalisation, thorough microbiological investigation and initiation of empirical broad-spectrum antipseudomonal antibiotic therapy, with rationalisation of antibiotic choice once susceptibility results of the isolate become available.

\section{References}

1. Greene SL, Su WP, Muller SA. Ecthyma gangrenosum: Report of clinical, histopathologic, and bacteriologic aspects of eight cases. J Am Acad Dermatol 1984;11(5 Pt 1):781-787.

2. Patel JK, Perez OA, Viera MH, Halem M, Berman B. Ecthyma gangrenosum caused by Escherichia coli bacteremia: A case report and review of the literature. Cutis 2009;84(5):261-267.

3. Peña C, Suarez C, Gozalo M, et al. Prospective multicenter study of the impact of carbapenem resistance on mortality in Pseudomonas aeruginosa bloodstream infections. Antimicrob Agents Chemother 2011;56(3):1265-1272. [http:// dx.doi.org/10.1128/AAC.05991-11]

4. Pechter PM, Marchione R, Milikowski C, Berman B. Ecthyma gangrenosum secondary to Staphylococcus aureus in an infant with transient neutropenia. Pediatr Dermatol 2012;29(3):320-323. [http:// dx.doi.org/10.1111/j.1525-1470.2011.01427.x]

5. Goolamali SI, Fogo A, Killian L, et al. Ecthyma gangrenosum: An important feature of pseudomonal sepsis in a previously well child Clin Exp Dermatol 2009;34(5):e180-e182. [http:// dx.doi.org/10.1111/j.1365-2230.2008.03020.x

6. Pandit AM, Siddaramappa B, Choudhary SV Manjunathswamy BS. Ecthyma gangrenosum in a new born child. Indian J Dermatol Venereo Leprol 2003;69(7):52-53.

7. Downey DM, O'Bryan MC, Burdette SD, Michael JR, Saxe JM. Ecthyma gangrenosum in a patient with toxic epidermal necrolysis. J Burn Care Res 2007;28(1):198-202. [http://dx.doi.org/10.1097/ BCR.0B013E31802CA481]

8. Fink M, Conrad D, Matthews M, Browning JC Primary ecthyma gangrenosum as a presenting sign in a child. Dermatol Online J 2012;18(3):3.

9. Smeets JG, Lowe SH, Veraart JC. Cutaneous infections with Stenotrophomonas maltophilia in patients using immunosuppressive medication. J Eur Acad Dermatol Venereol 2007;21(9):1298-1300. [http:// dx.doi.org/10.1111/j.1468-3083.2007.02201.x]

10. Marra AR, Pereira CA, Gales AC, et al. Bloodstream infections with metallo-beta-lactamase-producing Pseudomonas aeruginosa: Epidemiology, microbiology, and clinical outcomes. Antimicrob Agents Chemother 2006;50:388-390. 